\title{
Homomorphisms of Cayley Graphs and Cycle Double Covers
}

\author{
Radek Hušek Robert Š́mal* \\ Computer Science Institute \\ Charles University \\ Prague, Czech Republic \\ \{husek, samal\}@iuuk.mff.cuni.cz
}

Submitted: Jan 16, 2019; Accepted: Dec 31, 2019; Published: Apr 3, 2020

(c) The authors. Released under the CC BY license (International 4.0).

\begin{abstract}
We study the following conjecture of Matt DeVos: If there is a graph homomorphism from a Cayley graph Cay $(M, B)$ to another Cayley graph Cay $\left(M^{\prime}, B^{\prime}\right)$ then every graph with an $(M, B)$-flow has an $\left(M^{\prime}, B^{\prime}\right)$-flow. This conjecture was originally motivated by the flow-tension duality. We show that a natural strengthening of this conjecture does not hold in all cases but we conjecture that it still holds for an interesting subclass of them and we prove a partial result in this direction. We also show that the original conjecture implies the existence of an oriented cycle double cover with a small number of cycles.
\end{abstract}

Mathematics Subject Classifications: 05C60, 05C21, 05C15

\section{Introduction}

For an abelian group $M$ (all groups in this article are abelian even though we often do not say it explicitly), an $M$-flow $\varphi$ on a directed graph $G=(V, E)$ is a mapping $E \rightarrow M$ such that the oriented sum around every vertex $v$ is zero:

$$
\sum_{v w \in E} \varphi(v w)-\sum_{u v \in E} \varphi(u v)=0
$$

We say that $M$-flow $\varphi$ is an $(M, B)$-flow if $\varphi(e) \in B$ for all $e \in E$. We always assume that $B \subseteq M$ and that $B$ is symmetric, i. e., $B=-B$. This implies that reversing an edge $e$ and changing the sign of $\varphi(e)$ preserves the property of being an $(M, B)$-flow. Thus, the existence of such a flow depends only on the underlying undirected graph.

${ }^{*}$ Both authors were supported by GAČR grant 19-21082S. 
The main example is $B=M \backslash\{0\}$; in this case we call a $(M, B)$-flow a nowhere-zero $M$-flow. The study of nowhere-zero flows was started by Tutte [8, 7], main motivation was the fact, that a planar graph has a proper face $k$-coloring if and only if it has a nowhere-zero $\mathbb{Z}_{k}$-flow. For a more thorough introduction to the theory that came out of this we refer the reader to [3, Chapter 6] or [9]. Here we just present the results and notions crucial for our exposition.

Theorem 1 (Tutte [7]). Let $k \geqslant 2$ be an integer and let $f$ be a $\mathbb{Z}_{k}$-flow on a graph $G$. Then there is a $\mathbb{Z}$-flow $g$ on the same graph such that for every edge e we have $f(e) \equiv g(e)$ $(\bmod k)$ and $|g(e)|<k$. Conversely, for each $\mathbb{Z}$-flow $g$, the flow $f:=g \bmod k$ is a $\mathbb{Z}_{k}$-flow.

An $M$-tension $\tau$ on a digraph $G$ is again a mapping $E \rightarrow M$ but the condition is that the oriented sum along every cycle $C$ is zero, explicitly

$$
\sum_{e \in C^{+}} \tau(e)-\sum_{e \in C^{-}} \tau(e)=0
$$

where $C^{+}$are edges of $C$ with one orientation along the cycle and $C^{-}$the edges with the opposite orientation. We define $(M, B)$-tension to be an $M$-tension which uses only values from a symmetric set $B \subseteq M$.

Before stating our next observation, we need to define the notion of Cayley graph. Given an abelian group $M$ and its symmetric subset $B \subseteq M$ we let $\operatorname{Cay}(M, B)$ denote the graph with vertex set $M$ and with edges $\{u v: u, v \in M, v-u \in B\}$. The notion of a tension defined in the previous paragraph can be equivalently described by its relation to vertex colorings. Consider a mapping $p: V \rightarrow M$ (usually called a group coloring or in this context a potential). If we define $\tau(u v)$ as $p(v)-p(u)$ for every edge $u v$ (we write $\tau=\delta p$ ), then $\tau$ is a tension. On the other hand, it is easy to show that every tension can be written as $\delta p$ for some potential $p$. If $\tau$ is an $(M, B)$-tension, then $p$ only uses values in $B \subseteq M$, thus $p$ is a homomorphism into $\operatorname{Cay}(M, B)$.

For planar graphs, flows and tensions are dual notions - every flow in the primal graph corresponds to a tension in its dual and vice versa. Because a composition of homomorphisms is a homomorphism, the following statement holds:

Observation 2. Let $M, M^{\prime}$ be abelian groups and $B \subseteq M, B^{\prime} \subseteq M^{\prime}$ their symmetric subsets. If there is a graph homomorphism from $\operatorname{Cay}(M, B)$ into $\operatorname{Cay}\left(M^{\prime}, B^{\prime}\right)$, then every graph with an $(M, B)$-tension has an $\left(M^{\prime}, B^{\prime}\right)$-tension.

Many fruitful questions about flows on graphs were motivated by mimicking the properties of coloring in the dual setting [8]. In the same spirit, we ask for the dual version of Observation 2:

Conjecture 3 (DeVos [2]). Let $M, M^{\prime}$ be abelian groups and $B \subseteq M, B^{\prime} \subseteq M^{\prime}$ their symmetric subsets. If there is a graph homomorphism from $\operatorname{Cay}(M, B) \operatorname{into} \operatorname{Cay}\left(M^{\prime}, B^{\prime}\right)$, then every graph with an $(M, B)$-flow has an $\left(M^{\prime}, B^{\prime}\right)$-flow.

This is still an open problem but it holds in some special cases. We start with a few immediate observations that appear in [2]. The Conjecture 3 holds 
- if $G$ is planar (because of duality and Observation 2) or

- if $0 \in B^{\prime}$ (every graph has an $\left(M^{\prime},\{0\}\right)$-flow) or

- if $B=M \backslash\{0\}$ and $B^{\prime}=M^{\prime} \backslash\{0\}$ : Here an $(M, B)$-flow is just a nowhere-zero $M$-flow. It is known that the existence of a nowhere-zero flow is monotone in the size of the group [8].

A generalization of the last example is based on the monotonicity of circular flows. A circular $k / d$-flow is a $\mathbb{Z}$-flow $\varphi$ such that $d \leqslant|\varphi(e)| \leqslant k-d$ for every edge $e$. It was proved in Goddyn et al. [4] that every graph with a circular $k / d$-flow has a circular $k^{\prime} / d^{\prime}$-flow (assuming $k / d \leqslant k^{\prime} / d^{\prime}$ ).

Let $M=\mathbb{Z}_{k}, B= \pm\{d, d+1, \ldots, k-d\}, M^{\prime}=\mathbb{Z}_{k^{\prime}}, B^{\prime}= \pm\left\{d^{\prime}, d^{\prime}+1, \ldots, k^{\prime}-d^{\prime}\right\}$. Note that by Theorem 1 we may equivalently define circular $k / d$-flow to be a $\mathbb{Z}_{k}$-flow with values in $\{d, d+1, \ldots, k-d\}$, that is an $(M, B)$-flow. Thus the result of Goddyn et al. implies that every graph with $(M, B)$-flow has an $\left(M^{\prime}, B^{\prime}\right)$-flow if and only if $k / d \leqslant k^{\prime} / d^{\prime}$.

The Cayley graph $\operatorname{Cay}(M, B) \cong K_{k / d}$ is frequently denoted as the circular clique (also as circular complete graph); similarly, $\operatorname{Cay}\left(M^{\prime}, B^{\prime}\right) \cong K_{k^{\prime}} / d^{\prime}$. This graph is important in the study of circular coloring, the dual concept of circular flows. It is known that there is a homomorphism from $K_{k / d}$ to $K_{k^{\prime} / d^{\prime}}$ if and only if $k / d \leqslant k^{\prime} / d^{\prime}$ (see, for example, [11] or its references). Thus, both sides of the conjectured implication are in this setting equivalent to $k / d \leqslant k^{\prime} / d^{\prime}$, hence the conjecture holds for these combinations of groups and their subsets.

We thank the anonymous referee who kindly suggested that our example using circular $(2 k+1) / k$-flows extends to any two circular flows.

\section{New Framework}

The structure of homomorphisms from $\operatorname{Cay}(M, B)$ to $\operatorname{Cay}\left(M^{\prime}, B^{\prime}\right)$ is hard to describe. Instead we take any mapping $m: M \rightarrow M^{\prime}$ (not necessarily a group homomorphism) and let $B^{\prime}$ be determined by $m$ (so $B^{\prime}$ is the minimal set for which $m$ is a graph homomorphism). This is achieved by the following technical definition:

Definition 4. Let $M, M^{\prime}$ be abelian groups and $m: M \rightarrow M^{\prime}$ any mapping. For $x \in M$ we define its homomorphic image

$$
\mathcal{H}_{m}(x):=\{m(a+x)-m(a): a \in M\} .
$$

We omit the index $m$ whenever possible. Observe that in the case of tensions $\mathcal{H}(x)$ is exactly the set of possible images of value $x$ on some edge after composing original tension represented by a group coloring with $m$ :

Observation 5. Let $p: V \rightarrow M$ be a group coloring and let $m: M \rightarrow M^{\prime}$ be any mapping between abelian groups $M$ and $M^{\prime}$. Define $p^{\prime}=m \circ p, \tau=\delta p$, and $\tau^{\prime}=\delta p^{\prime}$. Then

- $\tau$ is a M-tension, 
- $\tau^{\prime}$ is a $M^{\prime}$-tension, and

- $\forall e \in E: \tau^{\prime}(e) \in \mathcal{H}(\tau(e))$.

Property 6 (Homomorphism property). Let $G$ be a (directed) graph and $m: M \rightarrow M^{\prime}$ an arbitrary mapping between abelian groups. We say that $G$ has homomorphism property (HP) for $m$ if for every $(M, B)$-flow there exists an $\left(M^{\prime}, \bigcup_{x \in B} \mathcal{H}(x)\right)$-flow. We say that $G$ has HP for group $M$ if it has HP for all mappings $m$ with domain $M$, and that $G$ has HP if it has HP for all abelian groups.

The name of the property is due to the fact that $m$ is a graph homomorphism from $\operatorname{Cay}\left(M, \bigcup_{e \in E} \varphi(e)\right)$ to $\operatorname{Cay}\left(M^{\prime}, \bigcup_{e \in E} \mathcal{H}(\varphi(e))\right)$.

Conjecture 7 (Reformulation of Conjecture 3). All graphs have homomorphism property.

Proof of equivalence of Conjectures 3 and 7 . For any mapping $m: M \rightarrow M^{\prime}$, we put $B^{\prime \prime}=\bigcup_{x \in B} \mathcal{H}(x)$. As $m$ is a homomorphism of Cay $(M, B)$ to $\operatorname{Cay}\left(M^{\prime}, B^{\prime \prime}\right)$, Conjecture 3 implies Conjecture 7. On the other hand, if $m$ is any homomorphism of $\operatorname{Cay}(M, B)$ to $\mathrm{Cay}\left(M^{\prime}, B^{\prime}\right)$ (note the different target graph), then $B^{\prime \prime} \subseteq B^{\prime}$. Consequently, every $\left(M^{\prime}, B^{\prime \prime}\right)$-flow is also an $\left(M^{\prime}, B^{\prime}\right)$-flow and thus Conjecture 7 implies Conjecture 3.

The traditional approach to solving flow-related conjectures is to study properties of a hypothetical minimal counterexample. Usually the problem is reduced to cubic graphs by splitting / decontracting vertices. This, however, is not possible with Conjecture 3 because decontracting a vertex may create an edge with a new value found nowhere else, modifying $B$. To overcome this we formulated the following property which is a strengthening of the homomorphism property:

Property 8 (Strong homomorphism property). Let $G$ be a (directed) graph and $m: M \rightarrow$ $M^{\prime}$ an arbitrary mapping between abelian groups. We say that $G$ has strong homomorphism property (SHP) for $m$ if for every $M$-flow $\varphi$ there exists an $M^{\prime}$-flow $\varphi^{\prime}$ such that $\varphi^{\prime}(e) \in \mathcal{H}(\varphi(e))$ for all edges $e$. We say that $G$ has SHP for group $M$ if it has SHP for all mappings $m$ with domain $M$, and that $G$ has SHP if it has SHP for all abelian groups.

Note that SHP allows the flow to be zero on some edges but such edges are not interesting because $\mathcal{H}(0)$ is always $\{0\}$. The SHP allows us to study only cubic graphs - we can make any graph (sub)cubic by decontracting its vertices of high degree and if SHP holds for such decontracted graphs then it holds for the original graph too. To state this in a formal way, we need the following technical definition:

Definition 9. We say that a digraph $H$ is a cubification of digraph $G$ if $H$ can be obtained from $G$ using following operations:

1. decontraction of vertex of degree at least 4 (such that both new vertices have degree at least 3),

2. suppression of a vertex of degree 2 , 
3. deletion of a bridge,

4. deletion of a loop, and

5. deletion of an isolated vertex.

With this definition we want to show that every non-cubic graph can be reduced to a smaller cubic one. To get this we need to use a slightly non-standard definition of the size of the graph which considers graphs with larger degrees bigger. Suitable definition for us is

$$
\Phi:=\sum_{v \in V} 3^{\operatorname{deg} v}
$$

Observation 10 (Reducibility of SHP to cubic graphs). Let $m: M \rightarrow M^{\prime}$ an arbitrary mapping between abelian groups and let $G$ be a digraph. If some cubification of $G$ has $S H P$ for $m$, then also $G$ has SHP for $m$. Moreover for every flow $\varphi: E \rightarrow M$ there exists a non-strictly smaller (possibly empty) cubic graph $G^{\prime}=\left(V^{\prime}, E^{\prime}\right)$ and a nowhere-zero flow $\varphi^{\prime}: E^{\prime} \rightarrow M$ such that if SHP does not hold for $\varphi$ on $G$ then SHP also does not hold for $\varphi^{\prime}$ on $G^{\prime}$.

Proof. To prove the first part, we only need to show that inverse of each operation used in Definition 9 does not break SHP:

1. Suppose $G=G_{1} / e$ and $G_{1}$ has SHP for $m$. Let $\varphi$ be an $M$-flow on $G$. There is a unique extension of $\varphi$ to $G_{1}$, we use $\varphi$ for this extension as well. (Note that the value of $\varphi(e)$ may be 0.) As $G_{1}$ has SHP for $M$, there is an $M^{\prime}$-flow $\varphi^{\prime}$ on $G_{1}$ such that $\varphi^{\prime}(e) \in \mathcal{H}_{m}(\varphi(e))$. The restriction of $\varphi^{\prime}$ to $G=G_{1} / e$ is the desired $M^{\prime}$-flow on $G$.

2. Subdivision of an edge is obvious when the new vertex of degree 2 has both in-degree and out-degree 1 . In the other case SHP still holds because $\mathcal{H}(-x)=-\mathcal{H}(x)$.

3. Addition of a bridge does not break SHP because flow on a bridge is always 0 and $\mathcal{H}(0)=\{0\}$.

4. Addition of a loop is also simple because $\mathcal{H}(x)$ is always non-empty and we can assign any value on a loop without affecting the rest of the flow.

5. Addition of an isolated vertex does not change the flow at all.

The moreover part: Note that $\Phi=\sum_{v \in V} 3^{\operatorname{deg} v}$ for every cubification is strictly smaller than $\Phi$ of the original graph. To obtain $G^{\prime}$ we set $G^{\prime}=G, \varphi^{\prime}=\varphi$, and apply the following operations as long as possible:

1. Remove an edge $e^{\prime} \in E^{\prime}$ such that $\varphi^{\prime}\left(e^{\prime}\right)=0$.

2. Apply some cubification operation on $G^{\prime}$. 
Because each of the operations decreases $\Phi\left(G^{\prime}\right)$, the process terminates. If the resulting $\varphi^{\prime}$ was not nowhere-zero, we still could remove an edge with 0 flow, and if $G^{\prime}$ was not cubic, we could get a non-trivial cubification.

The SHP is a natural strengthening of HP - we just fix a particular $(M, B)$-flow $\varphi$ and try to find an $\left(M^{\prime}, B^{\prime}\right)$-flow $\varphi^{\prime}$ with an extra requirement $\varphi^{\prime}(e) \in \mathcal{H}(\varphi(e))$. Observation 5 shows that a variation of SHP for tensions holds in general, so also all planar graphs have SHP due to duality.

With computer aid we found out that not all graphs have a SHP. The smallest graphs without SHP that we found are $K_{5}$ and $K_{3,3}$ with a particular $\mathbb{Z}_{5}$-flow and the universal mapping; see Figure 1 and Definition 13 below. Due to the universal mapping concept, and given the problematic $\mathbb{Z}_{5}$-flow, it is actually easy to see by hand that $K_{3,3}$ does not have SHP.

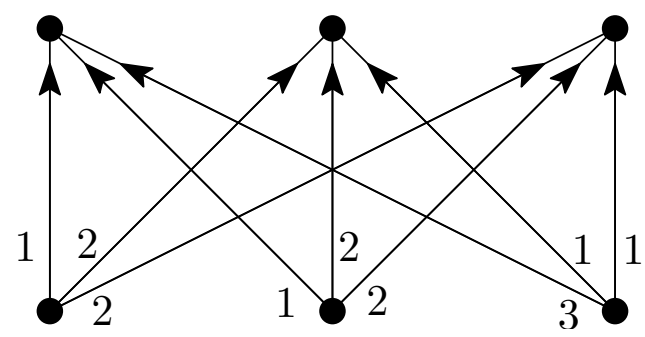

Figure 1: A graph with a $\mathbb{Z}_{5}$-flow for which SHP does not hold.

Although SHP does not hold for $K_{5}$ and $K_{3,3}$ in general it still holds for groups $\mathbb{Z}_{3}$ and $\mathbb{Z}_{4}$ because SHP always holds for groups of size at most 4 (Theorem 16). We also tested that SHP holds for Petersen graph and $\mathbb{Z}_{5}$ (we did not try larger snarks due to computational complexity). This motivates our next conjecture:

Conjecture 11 (SHP for minimal groups). For every graph $G$ the strong homomorphism property holds for group $\mathbb{Z}_{k}$ where $k$ is minimal such that $G$ admits a nowhere-zero $\mathbb{Z}_{k^{-}}$ flow.

It is easy to observe that SHP holds for $m$ which are (induced by) a group homomorphism but a more general statement is true:

Observation 12. Let $G$ be a graph and let $m: M \rightarrow M^{\prime}$ be some mapping of abelian groups. Let $h: M^{\prime} \rightarrow M^{\prime \prime}$ be a group homomorphism. If SHP (resp. HP) holds for $G$ and $m$ then it also holds for $G$ and $h \circ m$.

Proof. Let $\varphi^{\prime}$ be an $M^{\prime}$-flow guaranteed by SHP. For a group homomorphism $h$ it holds $\mathcal{H}_{h}(x)=\{h(x)\}$. Then $\varphi^{\prime \prime}=h \circ \varphi^{\prime}$ is also an $M^{\prime \prime}$-flow and its values satisfy

$$
\begin{aligned}
\varphi^{\prime \prime}(e) \in \mathcal{H}_{h}\left[\mathcal{H}_{m}(\varphi(e))\right] & =\{h(m(a+\varphi(e))-m(a)): a \in M\} \\
& =\{h(m(a+\varphi(e)))-h(m(a)): a \in M\}=\mathcal{H}_{h \circ m}(\varphi(e)) .
\end{aligned}
$$




\section{Universal objects}

Observation 12 leads us to the definition of a universal mapping such that if HP or SHP holds for this mapping, it also holds for every other mapping.

Definition 13 (Universal mapping). Let $M$ be an abelian group. We define its universal group $\mathcal{G}_{M}=\mathbb{Z}^{M}$ and its universal mapping $\mathcal{M}_{M}: M \rightarrow \mathcal{G}_{M}$ :

$$
x \longmapsto g_{x}
$$

where $g_{x}$ is a vector with 1 on position $x$ and 0 elsewhere.

The group $\mathbb{Z}^{M \backslash\{0\}}$ (with 0 mapped to 0 instead of $g_{0}$ ) would be sufficient but we choose the definition with $\mathbb{Z}^{M}$ to simplify the proofs. Note that with this definition $\mathcal{G}_{M}$ is just a free group generated by elements of $M$.

Observation 14. The universal mapping is universal for both HP and SHP, i. e., if for a given graph (and flow) HP (resp. SHP) holds for the universal mapping $\mathcal{M}_{M}$ then it holds for $M$.

Proof. Let $m: M \rightarrow M^{\prime}$ be any mapping. We can also interpret $m$ as a homomorphism $m_{\text {ext }}: \mathcal{G}_{M} \rightarrow M^{\prime}$ - mapping $m$ defines values of generators $e_{x}$ and hence it can be uniquely extended into mapping on the whole group which is a homomorphism (here we are using the fact that $k g_{x}=0 \Rightarrow k=0$ for $x \neq 0$ ). Moreover $m=m_{\text {ext }} \circ \mathcal{M}_{M}$ so Observation 12 finishes the proof.

There also exists a universal object on the left-hand side - a universal flow $\mathcal{F}$ (which is just the flow into a free group generated by edges outside of some fixed spanning tree) - but $\mathcal{G}_{\mathcal{F}}$ has infinitely many generators so we have not found any reasonable way to work with it. Also note that although the universal group is infinite, (S)HP holds for the universal group $\mathbb{Z}^{M}$ if and only if it holds for $\mathbb{Z}_{k}^{M}$ for any $k>\Delta(G)$.

\section{Partial results}

In this section we prove SHP for some special cases of the mapping or the group.

Theorem 15 (Mappings with one "hole"). Strong homomorphism property holds for mappings $m: \mathbb{Z}_{k} \rightarrow \mathbb{Z}_{l}$ defined by $m(x)=$ ax $\bmod l$ where $a \in \mathbb{Z}$. (Here we interpret elements of $\mathbb{Z}_{k}$ as integers $0,1,2, \ldots, k-1$.)

Proof. Note that mapping $m$ is a composition of mappings $m_{1}: \mathbb{Z}_{k} \rightarrow \mathbb{Z}$ defined by $m_{1}(x)=x$ and $m_{2}: \mathbb{Z} \rightarrow \mathbb{Z}_{l}$ defined by $m_{2}(x)=a x \bmod l$, and that $m_{2}$ is a group homomorphism. Hence we only need to show that SHP holds for $m_{1}$, the rest follows from Observation 12.

So we need to show that for every $\mathbb{Z}_{k}$-flow $\varphi$ there exists a $\mathbb{Z}$-flow $\varphi^{\prime}$ such that $\varphi^{\prime}(e) \in$ $\mathcal{H}_{m_{1}}(\varphi(e))=\{\varphi(e), \varphi(e)-k\}$. This, however, is a well-known result of Tutte [7]. 
Theorem 16. Strong homomorphism property holds for groups $\mathbb{Z}_{2}, \mathbb{Z}_{3}, \mathbb{Z}_{2}^{2}$, and $\mathbb{Z}_{4}$.

Proof. Due to Observation 10 we know that minimal counter-example is a cubic graph $G$ and nowhere-zero flow $\varphi$. We denote the generators of the right-hand side free group $a$, $b, c, \ldots$

- $\mathbb{Z}_{2}$ : The only graph cubic with nowhere-zero $\mathbb{Z}_{2}$-flow is the empty graph, for which the claim holds.

- $\mathbb{Z}_{3}$ : Let the mapping $m$ be $0 \mapsto a, 1 \mapsto b$, and $2 \mapsto c$. So $\mathcal{H}(1)=\{b-a, c-b, a-c\}$. A cubic graph has a nowhere-zero $\mathbb{Z}_{3}$-flow if and only if it is bipartite. (To see this, notice that by changing the orientation of the edges we may assume all flow-values are equal to 1, thus the vertices are either sources or sinks and no two sinks (neither two sources) can be connected by an arc.) So we make all edges directed from one partition to the other and split them into 3 perfect matchings. Observe that either $\varphi \equiv 1$ or $\varphi \equiv 2$ in which case we flip the orientation of edges to get the $\varphi \equiv 1$. We assign one of the following flow values to each matching: $b-a, c-b, a-c$.

- $\mathbb{Z}_{2}^{2}$ : Let the mapping $m$ be $00 \mapsto a, 01 \mapsto b, 10 \mapsto c$, and $11 \mapsto d$. Then

$$
\begin{aligned}
& \mathcal{H}(01)=\{ \pm(a-b), \pm(c-d)\}, \\
& \mathcal{H}(10)=\{ \pm(a-c), \pm(b-d)\} \\
& \mathcal{H}(11)=\{ \pm(a-d), \pm(b-c)\}
\end{aligned}
$$

Let $C_{1}, C_{2}, C_{3}: E \rightarrow\{0, \pm 1\}$ be integer flows on $G$ satisfying the following relations.

$$
\begin{aligned}
& C_{1}(e)=0 \Leftrightarrow \varphi(e)=01, \\
& C_{2}(e)=0 \Leftrightarrow \varphi(e)=10, \\
& C_{3}(e)=0 \Leftrightarrow \varphi(e)=11 .
\end{aligned}
$$

That is, the support of $C_{1}$ is the collection of circuits formed by edges $e$ with $\varphi(e) \in$ $\{10,11\}$; the signs are chosen arbitrary but consistently around each of the circuits. (Similarly for $C_{2}, C_{3}$.) We define $\psi: E \rightarrow \mathbb{Z}^{4}$ (recall that $a=(1,0,0,0) \in \mathbb{Z}^{4}$, and $b, c, d$ are defined similarly):

$$
\psi=\frac{C_{1}+C_{2}+C_{3}}{2} a+\frac{C_{1}-C_{2}-C_{3}}{2} b+\frac{-C_{1}+C_{2}-C_{3}}{2} c+\frac{-C_{1}-C_{2}+C_{3}}{2} d .
$$

It is easy to check that $\psi$ is a $\mathbb{Z}^{4}$-flow and $\psi(e) \in \mathcal{H}(\varphi(e))$.

- $\mathbb{Z}_{4}$ : We observe that every vertex (with all incident edges in the same direction) has either values $2,1,1$ or $2,3,3$. Hence edges with value 2 are a perfect matching. When we remove them we obtain disjoint union of circuits and we modify orientation of remaining edges so they are directed along circuits. With this orientation values around every vertex are 1,2,3 so both edges with value 1 and edges with value 3 are a perfect matching. 
Let $m$ be $0 \mapsto a, 1 \mapsto b, 2 \mapsto c$, and $3 \mapsto d$. Then

$$
\begin{aligned}
& \mathcal{H}(1)=\{b-a, c-b, d-c, a-d\}, \\
& \mathcal{H}(2)=\{ \pm(c-a), \pm(d-b)\}, \\
& \mathcal{H}(3)=\{d-a, a-b, b-c, c-d\} .
\end{aligned}
$$

Let $C_{1}, C_{2}, C_{3}: E \rightarrow\{0, \pm 1\}$ be a 3 -CDC of $G$ defined:

$$
\begin{aligned}
& e \in C_{1} \Leftrightarrow \varphi(e) \neq 1, \\
& e \in C_{2} \Leftrightarrow \varphi(e) \neq 2, \\
& e \in C_{3} \Leftrightarrow \varphi(e) \neq 3 .
\end{aligned}
$$

Observe that we can choose orientation of $C_{2}$ such that no edge has value -1 in $C_{2}$. And we define $\psi: E \rightarrow \mathbb{Z}^{4}$ (recall that $a=(1,0,0,0) \in \mathbb{Z}^{4}$, and $b, c, d$ are defined similarly):

$$
\psi=\frac{-C_{1}+C_{2}-C_{3}}{2} a+\frac{C_{1}-C_{2}-C_{3}}{2} b+\frac{C_{1}+C_{2}+C_{3}}{2} c+\frac{-C_{1}-C_{2}+C_{3}}{2} d .
$$

It is easy to check that $\psi$ is a $\mathbb{Z}^{4}$-flow and $\psi(e) \in \mathcal{H}(\varphi(e))$.

\section{Connection to CDC}

As we show below, flows obtained from HP or SHP can be easily transformed into an oriented cycle double cover. This is a collection of cycles, such that each edge is covered once in each direction, and each cycle is a collection of edge-disjoint circuits (with an assumed orientation). It was conjectured $[1,5]$ that such a collection always exists, see also [10]. This is one of the major open questions in the study of cycle spaces in graphs. Moreover if $G$ has SHP for $M$ and a nowhere-zero $M$-flow then the obtained CDC is orientable and has only $|M|$ cycles. This increases importance of Conjecture 7 and of determining for which graphs and groups does SHP hold.

Theorem 17 (Universal group and CDC). Let $M$ be any abelian group. If a graph $G$ has a flow in $\mathcal{G}_{M}$ using only values $\bigcup_{x \in M \backslash\{0\}} \mathcal{H}_{\mathcal{M}_{M}}(x)$ then it has an orientable cycle double cover using $|M|$ cycles.

Proof. Denote $H=\bigcup_{x \in M \backslash\{0\}} \mathcal{H}(x)$. Observe that all elements of $H$ are of form $g_{a}-g_{b}$ for some $a, b \in M$ and those $a, b$ are unique. Fix an $H$-flow $\varphi$. We define directed cycles (as mappings $E \rightarrow\{-1,0,1\}) \mathcal{C}_{x}(e):=(\varphi(e))_{x}$ and claim that $C=\left\{\mathcal{C}_{x}\right\}_{x \in M}$ is an orientable cycle double cover. From definition $C$ covers each edge twice, once in each direction, and every $\mathcal{C}_{x}$ is a flow with values $\{-1,0,1\}$ because it is a composition of a flow and group homomorphism so it is a cycle.

Corollary 18. If a graph has HP with respect to $\mathbb{Z}_{k}$ and nowhere-zero $\mathbb{Z}_{k}$-flow, then it has an orientable cycle double cover with at most $k$ cycles. 
Seymour in 1981 [6] proved that every graph without a bridge admits a nowhere-zero $\mathbb{Z}_{6}$-flow which combined with the previous corollary for $\mathbb{Z}_{6}$ gives us the following corollary.

Corollary 19. Conjecture 3 (and equivalently Conjecture 7) implies that every bridgeless graph has an orientable cycle double cover with at most 6 cycles.

\section{References}

[1] D. Archdeacon. Face colorings of embedded graphs. J. Graph Theory, 8(3):387-398, 1984.

[2] M. DeVos. A homomorphism problem for flows. Open Problem Garden. http://www.openproblemgarden.org/op/a_homomorphism_problem_for_flows, [retrieved 2020-02-28].

[3] R. Diestel. Graph theory, volume 173 of Graduate Texts in Mathematics. Springer, Berlin, fifth edition, 2017.

[4] L. A. Goddyn, M. Tarsi, and C.-Q. Zhang. On $(k, d)$-colorings and fractional nowherezero flows. J. Graph Theory, 28(3):155-161, 1998.

[5] F. Jaeger. Nowhere-zero flow problems. Selected topics in graph theory, 3:71-95, 1988.

[6] P. D. Seymour. Nowhere-zero 6-flows. J. Combin. Theory Ser. B, 30(2):130-135, 1981.

[7] W. T. Tutte. On the imbedding of linear graphs in surfaces. Proc. London Math. Soc., s2-51(1):474-483, 1949.

[8] W. T. Tutte. A contribution to the theory of chromatic polynomials. Canadian J. Math., 6:80-91, 1954.

[9] C.-Q. Zhang. Integer flows and cycle covers of graphs, volume 205 of Monographs and Textbooks in Pure and Applied Mathematics. Marcel Dekker, New York, 1997.

[10] C.-Q. Zhang. Circuit Double Cover of Graphs. London Mathematical Society Lecture Note Series. Cambridge University Press, 2012.

[11] X. Zhu. Circular chromatic number: a survey. Discrete Mathematics, 229(1-3):371410, 2001. 